\title{
DESINGULARIZATIONS OF PLANE VECTOR FIELDS
}

\author{
F. CANO
}

\begin{abstract}
The singularities of a plane vector field can be reduced under quadratic blowing ups. We describe a control method for the singularities of the vector field which works for ground fields of any characteristic and which has no essential obstruction for generalizing to higher dimensional cases.
\end{abstract}

0. Introduction. In his paper [6], Seidenberg proves the following result: "Let $k$ be an algebraically closed field of characteristic zero and let $D=A \partial / \partial X+B \partial / \partial Y$ be a vector field with $A, B \in k[[X, Y]]$ relatively prime elements. If $r=$ $\min (\nu(A), \nu(B))$, where $\nu$ denotes the order of the power series, then the solutions of $D$ can be made to go over the solutions of a finite number of vector fields with $r \leqslant 1$, by a finite number of transformations of the form $X^{\prime}=X, Y^{\prime}=Y / X$, translations tacitly included."

After this, A. van den Essen [7] gave another simpler proof of the same result based on the use of intersection multiplicities.

The above result may be seen as a "punctual" reduction of the singularities of the vector field $D$. In his works [2,3], Giraud proves implicitly a result (which may be stated as Corollary 2 below) on "global" reduction of the singularities of the vector field $D$, also valid for positive characteristic. Giraud considers vector fields that are tangents to a normal-crossing divisor and he uses

$$
c=\operatorname{length}(k[[X, Y]] /(A, B))
$$

as a main invariant for the control of the singularity, $A, B$ being the coefficients.

Giraud's invariant and Seidenberg's proof depend essentially on the fact that $A=B=0$ defines a scheme of dimension zero. So they are not generalizable to higher dimension.

In this paper we describe a control method for the singularity of the vector field based upon Hironaka's techniques in [4], which has no essential obstruction for generalizing to higher dimension (we have shown punctually in dimension 3 in [1]). We obtain a global result (Corollary 2 below) which is valid for all the characteristics. In $\$ 4$ we apply this technique to the case for which the minimum of the order of the coefficients would be 1 (see also [2]); in this case one cannot make a global reduction, but it is always possible to make a punctual reduction.

Received by the editors April 16, 1984 and, in revised form July 22, 1985.

1980 Mathematics Subject Classification. Primary 14D05, 14B05, 14E15.

Key words and phrases. Vector field, reduction of singularities. 


\section{Preliminaries.}

(1.1) Let $X$ be an excellent regular scheme of dimension two over an algebraically closed field $k$ of arbitrary characteristic. Let $E$ be a reduced effective divisor of $X$ whose support has only normal crossings as singularities and let us denote by $\Theta_{X}[E]$ the sub- $\mathcal{O}_{X}$-module of the tangent sheaf $\Theta_{X}$ locally given by the germs of vector fields which are germs of tangent vector fields to $E$.

(1.2) For any invertible sub- $\mathcal{O}_{X}$-module $\mathscr{D}$ of $\Theta_{X}$, let us denote by $\alpha(\mathscr{D})$ the invertible sub- $\mathcal{O}_{X}$-module of $\Theta_{X}$ which is locally the double orthogonal (i.e. the $\mathrm{D}^{\perp \perp}$ ) of $\mathscr{D}$ relative to the natural pairing $\Omega_{X} \times \Theta_{X} \rightarrow \mathcal{O}_{X}$. Let us denote by $(\mathscr{D}, E)$ the invertible sub- $\mathcal{O}_{X}$-module of $\Theta_{X}[E]$ given by $(\mathscr{D}, E)=\mathscr{D} \cap \Theta_{X}[E]$. If $\mathscr{D}=$ $(\alpha(\mathscr{D}), E)$, the sub- $\mathcal{O}_{X}$-module $\mathscr{D}$ of $\Theta_{X}[E]$ will be said to be multiplicatively irreducible and adapted to $E$.

(1.3) Let $(x, y)$ be a regular set of parameters for the local ring $\mathcal{O}_{X . P}$ of $X$ at $P$. Let us suppose that either $P \notin E$ or the local equations for $E$ at $P$ are $x=0, y=0$ or $x y=0$. Such a regular set of parameters will be called an adapted to $E$ regular set of parameters.

Let $\{\partial / \partial x, \partial / \partial y\}$ be the dual basis of $\{d x, d y\}$. Then the fiber $\Theta_{X, P}[E]$ of $\Theta_{X}[E]$ at $P$ is a free module over $\mathcal{O}_{X, P}$ which is freely generated by $\left\{\partial_{x}, \partial_{y}\right\}$, where $\partial_{x}=\partial / \partial x, \partial_{y}=\partial / \partial y$ if $P \notin E ; \quad \partial_{x}=x \cdot \partial / \partial x, \partial_{y}=\partial / \partial y$ if $E$ has the local equation $x=0$ at $P ; \partial_{x}=\partial / \partial x, \partial_{y}=y \cdot \partial / \partial y$ if $E$ has the local equation $y=0$ at $P$, and finally $\partial_{x}=x \cdot \partial / \partial x, \partial_{y}=y \cdot \partial / \partial y$ if $E$ has the local equation $x y=0$ at $P$.

A necessary and sufficient condition for $\mathscr{D}$ to be multiplicatively irreducible and adapted to $E$ is that for each closed point $P$, the fiber $\mathscr{D}_{P}$ would be generated by a germ of a vector field $D=a \partial_{x}+b \partial_{y}, a$ and $b$ being relatively prime elements in $\mathcal{O}_{X, P}$.

Throughout the paper we will suppose that $\mathscr{D}$ is multiplicatively irreducible and adapted to $E$.

(1.4) Consider $\pi: X^{\sim} \rightarrow X$, the blowing-up of $X$ with center in a closed point $P$. Let $\mathscr{D}^{\prime}$ denote the image of $\mathscr{D}$ by the composition of the two natural morphisms

$$
\Theta_{X} \rightarrow \operatorname{Hom}_{\mathcal{O}_{X}}\left(\Omega_{X}, \pi_{*} \mathcal{O}_{X^{-}}\right) \rightarrow \pi_{*} \operatorname{Hom}_{\mathcal{O}_{X-}}\left(\pi^{*} \Omega_{X}, \mathcal{O}_{X^{-}}\right)
$$

(the second one being an isomorphism) and let $\mathscr{D}^{\prime \prime}$ denote the image of the composition of the two natural morphisms

$$
\pi^{*} \mathscr{D}^{\prime} \rightarrow \pi^{*} \pi_{*} \operatorname{Hom}_{\mathcal{O}_{X-}}\left(\pi^{*} \Omega_{X}, \mathcal{O}_{X^{-}}\right) \rightarrow \operatorname{Hom}_{\mathcal{O}_{X-}}\left(\pi^{*} \Omega_{X}, \mathcal{O}_{X^{-}}\right)
$$

Finally, denote by $\mathscr{D}^{\pi}$ the inverse image of $\mathscr{D}^{\prime \prime}$ by the natural morphism

$$
\Theta_{X^{-}} \rightarrow \operatorname{Hom}_{\mathcal{O}_{X^{-}}}\left(\pi^{*} \Omega_{X}, \mathcal{O}_{X^{-}}\right) .
$$

$\mathscr{D}^{\pi}$ is an invertible sub- $\mathcal{O}_{X^{-}}$-module of $\Theta_{X^{-}}$. Let $E^{\pi}$ be the divisor of $X^{-}$(with normal crossings) obtained by considering on $\pi^{-1}(E \cup\{P\})$ its reduced structure. Define the adapted to E strict transform $\mathscr{D}^{-}$of $\mathscr{D}$ by $\pi$ to be $\mathscr{D}^{-}=\left(\alpha\left(\mathscr{D}^{\pi}\right), E^{\pi}\right)$.

(1.5) In terms of coordinates, $\mathscr{D}^{\sim}$ can be expressed as follows: Let $(x, y)$ be an adapted to $E$ regular set of parameters at the point $P$. For every closed point $P^{\prime}$ of $X^{\sim}$ with $\pi\left(P^{\prime}\right)=P$, an adapted to $E^{\pi}$ regular set of parameters $\left(x^{\prime}, y^{\prime}\right)$ can be 
obtained by exactly one of the two following transformations

$$
\begin{gathered}
x^{\prime}=x ; \quad x^{\prime}\left(y^{\prime}+\zeta\right)=y, \quad \zeta \in k, \\
x^{\prime} y^{\prime}=x ; \quad y^{\prime}=y .
\end{gathered}
$$

According to the notation in [4], the first one will be called a $(T-1, \zeta)$ transformation and the second one a $(T-2)$ transformation.

Suppose that we have a $(T-1, \zeta)$ transformation. If $P \notin E$ or if $x=0$ is a local equation for $E$ at $P$ or if $\zeta \neq 0$, then $x^{\prime}=0$ is a local equation for $E^{\pi}$ at $P^{\prime}$. If $\zeta=0$ and $y=0$ or $x y=0$ are local equations for $E$ at $P$, then $x^{\prime} y^{\prime}=0$ is a local equation for $E^{\pi}$ at $P^{\prime}$. Let $\mathscr{D}_{P}$ be generated by $D=a \partial_{x}+b \partial_{y}$. Then $\mathscr{D}_{P^{\prime}}$ is generated by the germ of the vector field $D^{\prime}$ given by

$$
D^{\prime}=x^{\prime t}\left(a x^{\prime-1} \partial_{x^{\prime}}+\left(b-\left(y^{\prime}+\zeta\right) a\right) x^{\prime-1} \partial_{y^{\prime}}\right)
$$

if $P \notin E$;

$$
D^{\prime}=x^{\prime t}\left(a \partial_{x^{\prime}}+\left(b x^{\prime-1}-\left(y^{\prime}+\zeta\right) a\right) \partial_{y^{\prime}}\right)
$$

if $x=0$ is a local equation for $E$ at $P$;

$$
D^{\prime}=x^{\prime \prime}\left(a x^{\prime-1} \partial_{x^{\prime}}+\left(b-a x^{\prime-1}\right) \partial_{y^{\prime}}\right)
$$

if $y=0$ is a local equation for $E$ at $P$ and $\zeta=0$;

$$
D^{\prime}=x^{\prime \prime}\left(a x^{\prime-1} \partial_{x^{\prime}}+\left(b-a x^{\prime-1}\right)\left(y^{\prime}+\zeta\right) \partial_{y^{\prime}}\right)
$$

if $y=0$ is a local equation for $E$ at $P$ and $\zeta \neq 0$;

$$
D^{\prime}=x^{\prime \prime}\left(a \partial_{x^{\prime}}+(b-a) \partial_{y^{\prime}}\right)
$$

if $x y=0$ is a local equation for $E$ at $P$ and $\zeta=0$; and

$$
D^{\prime}=x^{\prime t}\left(a \partial_{x^{\prime}}+(b-a)\left(y^{\prime}+\zeta\right) \partial_{y^{\prime}}\right)
$$

if $x y=0$ is a local equation for $E$ at $P$ and $\zeta \neq 0$. In the above expressions $t$ is the integer such that the coefficients of $\partial_{x^{\prime}}$ and $\partial_{y^{\prime}}$ in $D^{\prime}$ are relatively prime elements in the regular local ring $\mathcal{O}_{X^{-}, P^{\prime}}$

For a $T-2$ transformation, one can obtain analogous expressions for $D^{\prime}$. Namely, these expressions are those obtained by interchanging $x$ and $y, x^{\prime}$ and $y^{\prime}$ in the above cases for $\zeta=0$.

(1.6) The adapted order $\nu_{P}(\mathscr{D}, E)$ of $\mathscr{D}$ at a closed point $P$ of $X$ is defined to be the maximum integer $n$ such that

$$
\mathscr{D}_{P} \subseteq m_{P}^{n} \cdot \Theta_{X, P}[E],
$$

where $m_{P}$ is the maximal ideal of $\mathcal{O}_{X, P}$. The adapted order $\nu_{P}(\mathscr{D}, E)$ is also the minimum of the orders, relative to the filtration given by $m_{P}$ in $\mathcal{O}_{X, P}$, of the coefficients of any generator of $\mathscr{D}_{P}$ in any basis of $\Theta_{X, P}[E]$. If $\nu_{p}(\mathscr{D}, E)=0, P$ will be said to be a regular point for $\mathscr{D}$ adapted to $E$.

Looking at the expressions (1.5.3)-(1.5.8) it can be easily deduced that

$$
\nu_{P^{\prime}}\left(\mathscr{D}^{\sim}, E^{\pi}\right) \leqslant \nu_{P}(\mathscr{D}, E) \text {. }
$$




\section{Construction of the invariant.}

(2.1) Let us denote by $s_{P}(E)$ the number of irreducible components of $E$ through $P$. Let $(x, y)$ be an adapted to $E$ regular set of parameters at $P$ and let us suppose that $\mathscr{D}_{P}$ is generated by $D=a \partial_{x}+b \partial_{y}$. The point $P$ will be said to be of type zero for $\mathscr{D}$ if:

(i) $s_{p}(E)=1$.

(ii) The order $\nu(a)($ resp. $\nu(b))$ of $a$ (resp. $b$ ) relative to $m_{P}$ is equal to $\nu_{p}(\mathscr{D}, E)$ in the case that $y=0$ (resp. $x=0$ ) is a local equation for $E$ at $P$.

Otherwise, $P$ will be said to be of type one.

For an element $f \in \mathcal{O}_{X, P}$ with order $\nu(f) \geqslant r, r \in \mathbf{N}$, denote by $\mathscr{J}^{r}(f)$ the affine plane $\mathbf{A}^{2}(k)$ if $r<\nu(f)$ and the Hironaka tangent space (or directrix, see [4]) if $r=\nu(f)$ (i.e., in this case $\mathscr{J}^{r}(f)$ is the maximum linear subvariety of the tangent cone of $f=0$ leaving it invariant by translations).

Set $r=\nu_{P}(\mathscr{D}, E)$. We will define the directrix $\mathscr{J}_{P}(\mathscr{D}, E)$ of $\mathscr{D}$ at $P$ adapted to $E$ as either

$$
\mathscr{J}_{P}(\mathscr{D}, E)=\mathscr{J}^{r}(a) \cap \mathscr{J}^{r}(b)
$$

if $s_{P}(E)=0$ or 2 , or

$$
\mathscr{J}_{P}(\mathscr{D}, E)=\mathscr{J}^{r}(a)
$$

if $P$ is of type zero (resp. one) and $y=0$ (resp. $x=0$ ) is a local equation for $E$ at $P$, or finally

$$
\mathscr{J}_{P}(\mathscr{D}, E)=\mathscr{J}^{r}(b)
$$

if $P$ is of type zero (resp. one) and $x=0$ (resp. $y=0$ ) is a local equation for $E$ at $P$.

We always have that $\operatorname{dim} \mathscr{J}_{P}(\mathscr{D}, E) \leqslant 1$. If $\operatorname{dim} \mathscr{J}_{P}(\mathscr{D}, E)=1$, the directrix defines a closed point $\mathbf{P}\left(\mathscr{J}_{P}(\mathscr{D}, E)\right)$ in the exceptional divisor of the blowing-up $\pi: X^{\sim} \rightarrow X$ of $X$ with center $P$.

(2.2) LEMMA 1. If $P^{\prime}$ is a closed point of the exceptional divisor of $\pi$ such that $\nu_{P^{\prime}}\left(\mathscr{D}^{\sim}, E^{\pi}\right)=\nu_{P}(\mathscr{D}, E)$, then $\operatorname{dim} \mathscr{J}_{P}(\mathscr{D}, E)=1$ and $P^{\prime}=\mathbf{P}\left(\mathscr{J}_{P}(\mathscr{D}, E)\right)$.

Proof. Let us suppose that $P$ is of the type zero and that $x=0$ is a local equation for $E$ at $P$. Set $r=\nu_{P}(\mathscr{D}, E)$ and let us suppose that we have a $(T-1, \zeta)$ transformation and that $\mathscr{D} \tilde{P^{\prime}}$ is generated by $D^{\prime}=a^{\prime} \partial_{x^{\prime}}+b^{\prime} \partial_{y^{\prime}}$. Looking at (1.5.4) one realizes that $t=-1(r-1)$ and that

$$
a^{\prime}=a x^{\prime-r+1} ; \quad b^{\prime}=b x^{-r}-\left(y^{\prime}+\zeta\right) a x^{\prime-r+1} .
$$

If $\nu^{\prime}$ denotes the order relative to $m_{P^{\prime}}, \nu_{P^{\prime}}\left(\mathscr{D}^{\sim}, E^{\pi}\right)=r$ implies that $\nu^{\prime}\left(b x^{\prime-r}\right)=r$ since the order $\nu(b)$ of $b$ relative to $m_{P}$ is $r$. Let $\phi$ denote the initial form of $b$. A necessary condition in order to have $\nu^{\prime}\left(b x^{\prime-r}\right)=r$ is that one has $\phi=\lambda(\underline{y}-\zeta \underline{x})$, $\lambda \in k^{*}$, where $\underline{x}$ (resp. $y$ ) is the initial form of $x$ (resp. $y$ ), and in this case $\mathscr{J}^{r}(b)=(\underline{y}-\zeta \underline{x}=0)=\overline{\mathscr{J}}_{P}(\mathscr{D}, E)$. If $\operatorname{dim} \mathscr{J}_{P}(\mathscr{D}, E)=0$, then $\phi$ is not a power of a linear form and $\nu_{P^{\prime}}\left(\mathscr{D}^{\sim}, E^{\pi}\right)<r$.

A similar argument can be considered for $T-2$ and in the other cases. 
(2.3) Let us suppose that $s_{p}(E) \geqslant 1$ and $\operatorname{dim} \mathscr{J}_{P}(\mathscr{D}, E)=1$. By making, if necessary, changes of type $x_{1}=y$ or $y_{1}=y+\lambda x, \lambda \in k$, in an adapted to $E$ regular set of parameters $(x, y)$ one can assume that $(x, y)$ can be chosen such that a generator $D=a \partial_{x}+b \partial_{y}$ of $\mathscr{D}_{P}$ takes exactly one of the following forms:

I. $E$ given by $x$, type zero, $\mathscr{J}^{r}(b)=(\underline{y}=0)$.

II. $E$ given by $y$, type zero, $\mathscr{J}^{r}(a)=(\underline{y}=0)$.

III. $E$ given by $x$, type one, $\mathscr{J}^{r}(a)=(\underline{y}=0)$.

IV. $E$ given by $y$, type one, $\mathscr{J}^{r}(b)=(\underline{y}=0)$.

V. $E$ given by $x y, \quad \mathscr{J}_{P}(\mathscr{D}, E)=(\underline{y}+\zeta \underline{x}=0), \quad \zeta \in k$.

If the forms are I or III, we define the stability invariant $\mathrm{st}_{P}(\mathscr{D}, E)$ to be 0 and if

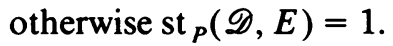

Following notations as in [4], for a power series $f=\sum_{i, j} f_{i j} x^{i} y^{i} \in k[[x, y]]$ and $r \in \mathbf{N}$, set

$$
\gamma^{r}(f ; x, y)=\min \left(i(r-j)^{-1} ; j<r, f_{i j} \neq 0\right) .
$$

If $r=$ order of $f,\left(x^{\prime}, y^{\prime}\right)$ are given by a $(T-1,0)$ transformation and $f^{\prime}=f \cdot x^{\prime-r}$, one has that

$$
\gamma^{r}\left(f^{\prime} ; x^{\prime}, y^{\prime}\right)=\gamma^{r}(f ; x, y)-1 \text {. }
$$

Moreover, the necessary and sufficient condition for $f$ to have order $<r$ is that $\gamma^{r}(f ; x, y)<1$, and if $r=\operatorname{order}$ of $f$, then $\mathscr{J}^{r}(f)=(\underline{y}=0)$ if and only if $\gamma^{r}(f ; x, y)>1$.

Set $r=\nu_{P}(\mathscr{D}, E)$. We define $\gamma(\mathscr{D}, E ; x, y)$ to be equal to

$$
\begin{aligned}
& \min \left(\gamma^{r}(y a ; x, y), \gamma^{r}(b ; x, y)\right), \text { if I, } \\
& \min \left(\gamma^{r}(a ; x, y), \gamma^{r}(y b ; x, y)\right), \text { if II, } \\
& \min \left(\gamma^{r+1}(y a ; x, y), \gamma^{r+1}(b ; x, y)\right), \text { if III, } \\
& \min \left(\gamma^{r+1}(a ; x, y), \gamma^{r+1}(y b ; x, y)\right), \text { if IV, } \\
& \min \left(\gamma^{r}(a ; x, y), \gamma^{r}(b ; x, y)\right), \text { if V. }
\end{aligned}
$$

Since $a$ and $b$ are relatively prime, one has that $\gamma_{P}(\mathscr{D}, E ; x, y)<\infty$ if $r \geqslant 2$.

$\mathscr{D}$ will be said to be prepared with respect to $(x, y)$ if one of the following situations holds:

(i) $\gamma=\gamma(\mathscr{D}, E ; x, y) \notin \mathbf{N}$.

(ii) $\gamma \in \mathbf{N}$ and $\mathscr{D}_{P}$ takes one of the forms II, IV, or V.

(iii) $\gamma \in \mathbf{N}$ and $\gamma$ does not increase after any change of the type $y_{1}=y+\lambda x^{\gamma}$, $\lambda \in k$.

If $r \geqslant 2$ and $\mathscr{D}$ is not prepared with respect to $(x, y)$, then from $(x, y)$ one can obtain a new adapted to $E$ regular set of parameters $\left(x, y^{\sim}\right)$ such that $\mathscr{D}$ is prepared with respect to $\left(x, y^{\sim}\right)$ by making successive changes of the type $y_{1}=y+$ $\lambda x^{\gamma}$ increasing $\gamma=\gamma(\mathscr{D}, E ; x, y)$. The number of these changes is always finite since otherwise $a$ and $b$ would not be relatively prime elements in $\mathcal{O}_{X, P}$ ( $a$ and $b$ are 
relatively prime in $\mathcal{O}_{X, P}$ if and only if they are relatively prime in the completion $\left.\hat{\mathcal{O}_{X, P}}\right)$. If $r=1$ it may happen that one has to make infinitely many changes $y \mapsto y_{1}$ (see Example (4.5) below).

Now, we define $\gamma_{P}(\mathscr{D}, E)$ to be the minimum $\gamma_{P}(\mathscr{D}, E ; x, y)$, where $(x, y)$ runs over all prepared situations. If $r=1$ and if the number of changes $y \mapsto y_{1}$ needed from any adapted to $E$ regular set of parameters $(x, y)$ to reach a prepared situation is infinite or if for any adapted to $E$ regular set of parameters $(x, y)$ with respect to which $\mathscr{D}$ is prepared we have that $\gamma(\mathscr{D}, E ; x, y)=\infty$, then we define $\gamma_{P}(\mathscr{D}, E)=\infty$. For the sake of completeness, we make the convention $\gamma_{P}(\mathscr{D}, E)=0$ if $s_{P}(E)=0$ or $\operatorname{dim} \mathscr{J}_{P}(\mathscr{D}, E)=0$.

(2.4) By Lemma 1, we can obtain by successive blowing-ups only one sequence

$$
P=P_{0} \stackrel{\pi_{0}}{\leftarrow} P_{1} \stackrel{\pi_{1}}{\leftarrow} \cdots,
$$

each $P_{i}$ being the center of $\pi_{i}$ and lying in the exceptional divisor of $\pi_{i-1}$, in such a way that the adapted order of the successive adapted to $E_{i-1}$ strict transforms $\mathscr{D}_{i}$ of $\mathscr{D}_{i-1}$ does not change $\left(\mathscr{D}_{0}=\mathscr{D}, E_{0}=E\right)$. This sequence will be called the adapted to $E$ stationary way of $P$ for $\mathscr{D}$ and it will be denoted by $S_{P}(\mathscr{D}, E)$. The length $l_{P}(\mathscr{D}, E)$ of $S_{P}(\mathscr{D}, E)$ may be "a priori" finite or infinite.

Let $i \leqslant l_{P}(\mathscr{D}, E)$. We define the number $\delta_{P}\left(\mathscr{D}, E ; P_{i}\right)$ to be equal to 1 if $i=0$ and equal to 0 if $i>0$.

Now, we define the (lexicographic) invariant $\operatorname{Inv}_{P}\left(\mathscr{D}, E ; P_{i}\right)$ as

$$
\begin{aligned}
\operatorname{Inv}_{P}\left(\mathscr{D}, E ; P_{i}\right)=\left(\operatorname{dim} \mathscr{J}_{P_{i}}\left(\mathscr{D}_{i}, E_{i}\right), \delta_{P}\left(\mathscr{D}, E ; P_{i}\right), \text { type of } P_{i},\right. \\
\left.\operatorname{st}_{P_{i}}\left(\mathscr{D}_{i}, E_{i}\right), \gamma_{P_{i}}\left(\mathscr{D}_{i}, E_{i}\right)\right) .
\end{aligned}
$$

\section{Desingularization results (order $\geqslant 2$ ).}

(3.1) Lemma 2. Let $0 \leqslant i \leqslant l_{P}(\mathscr{D}, E)$ and let us suppose that $\mathscr{D}_{i}$ takes at $P_{i}$ the form I (resp. III, resp. IV, resp. V) and that $\mathscr{D}_{i+1}$ takes at $P_{i+1}$ the form I (resp. III, resp. V, resp. V). Then one has that

$$
\gamma_{P_{i+1}}\left(\mathscr{D}_{i+1}, E_{i+1}\right) \leqslant \gamma_{P_{i}}\left(\mathscr{D}_{i}, E_{i}\right)-1 \text {. }
$$

Proof. If $\gamma_{P_{i}}\left(\mathscr{D}_{i}, E_{i}\right)=\infty$, there is nothing to see. Suppose that $\gamma_{P_{i}}\left(\mathscr{D}_{i}, E_{i}\right)<\infty$. If $(x, y)$ is an adapted to $E_{i}$ regular set of parameters with respect to which $\mathscr{D}_{i}$ is prepared at $P_{i}$ and for which $\mathscr{D}_{i}$ takes the form I, III, IV or V, then the only transformation to make is $(T-1,0)$ (note that in case $\mathrm{V}$ one necessarily has $\zeta=0$, since otherwise one would not have the form $V$ in $\mathscr{D}_{i+1}$ ). Because of (2.3.3) one can see that

$$
\gamma\left(\mathscr{D}_{i+1}, E_{i+1} ; x^{\prime}, y^{\prime}\right)=\gamma\left(\mathscr{D}_{i}, E_{i} ; x, y\right)-1 .
$$

Now, in view of the commutativity of making the change $y_{1}^{\prime}=y^{\prime}+\lambda x^{\prime \gamma^{\prime}}$ after $(T-1,0)$ or making $(T-1,0)$ after the change $y_{1}=y+\lambda x^{\gamma}$, with $\gamma=\gamma^{\prime}+1$, one has that $\mathscr{D}_{i+1}$ is prepared with respect to $\left(x^{\prime}, y^{\prime}\right)$ and (3.1.1) follows from this fact. 
(3.2) Lemma 3. Let $0<i \leqslant l_{P}(\mathscr{D}, E)$ and let us suppose that $\mathscr{D}_{i}$ takes the form II at $P_{i}$. Then we have that $i=l_{P}(\mathscr{D}, E)$ if $r=\nu_{P}(\mathscr{D}, E) \geqslant 2$. If $r=1$ and $i<l_{P}(\mathscr{D}, E)$, then we have that

$$
\operatorname{dim} \mathscr{J}_{P_{i+1}}\left(\mathscr{D}_{i+1}, E_{i+1}\right)=0 .
$$

REMARK. If we have (3.2.1), then $i+1=l_{P}(\mathscr{D}, E)$ by Lemma 1 .

PROOF OF LEMMA 3. By the above remark, $\operatorname{dim} \mathscr{J}_{P_{i-1}}\left(\mathscr{D}_{i-1}, E_{i-1}\right)=1$. If $s_{P_{t-1}}\left(E_{i-1}\right) \geqslant 1$, then $\mathscr{D}_{i-1}$ takes at $P_{i-1}$ one of the forms III or $\mathrm{V}$ (the other forms cannot be transformed into II).

Let us suppose that $\mathscr{D}_{i-1}$ has the form III and that it is generated at $P_{i-1}$ by

$$
a(x \partial / \partial x)+b \partial / \partial y
$$

with order of $b \geqslant r+1$ and $\mathscr{J}^{r}(a)=(\underline{y}=0)$. If we apply $(T-1,0)$, then $\mathscr{D}_{i}$ is generated at $P_{i}$ by

$$
a^{\prime}\left(x^{\prime} \partial / \partial x^{\prime}\right)+b^{\prime} \partial / \partial y^{\prime}
$$

where $a^{\prime}=a / x^{\prime r}, b^{\prime}=b / x^{\prime r+1}-y^{\prime} a / x^{\prime r}$. Because of our hypotheses on the form II of $\mathscr{D}_{i}$ at $P_{i}$, we have that

(3.2.4) $\quad a^{\prime}=\lambda y^{\prime r}+x^{\prime}(\cdots), \quad b^{\prime}=\mu x^{\prime r}+($ terms of higher order $)$

with $\lambda, \mu \in k^{*}=k-\{0\}$. Interchanging $x^{\prime}$ and $y^{\prime}$, we can suppose that $\mathscr{D}_{i}$ is generated at $P_{i}$ by

$$
\left(\mu y^{r}+(\cdots)\right) \partial / \partial x+\left(\lambda x^{r}+y(\cdots)\right)(y \partial / \partial y) .
$$

By applying $(T-1,0)$ to (3.2.5) one can see that the adapted order drops if $r \geqslant 2$, so $i=l_{P}(\mathscr{D}, E)$, and that the dimension of the directrix drops if $r=1$ and if the adapted order does not drop, so we have (3.2.1).

Let us suppose that $\mathscr{D}_{i-1}$ has the form $\mathrm{V}$ and that it is generated at $P_{i-1}$ by

$$
a(x \partial / \partial x)+b(y \partial / \partial y)
$$

where $\mathscr{J}^{r}(a) \cap \mathscr{J}^{r}(b)=(\underline{y}-\zeta \underline{x}=0)$ (necessarily $\zeta \neq 0$, otherwise $\left.s_{P_{i}}\left(E_{i}\right)=2\right)$. If we apply $(T-1,0)$, then $\mathscr{D}_{i}$ is generated at $P_{i}$ by (3.2.3) where $a^{\prime}=a / x^{\prime r}$ and $b^{\prime}=(b-a)\left(y^{\prime}+\zeta\right) / x^{\prime r}$. By the hypotheses, one has necessarily $\operatorname{In}\left(b^{\prime}\right)=\mu \underline{x}^{\prime r}$, $\mu \neq 0$, so $\operatorname{In}(a)=\operatorname{In}(b)=\lambda(\underline{y}-\zeta \underline{x})^{r}, \lambda \neq 0$, since otherwise $\underline{y}^{r}$ will occur in $\operatorname{In}\left(b^{\prime}\right)$. Thus we will have (3.2.4) and the same conclusions as in the preceding case.

Now suppose that $s_{P_{i-1}}\left(E_{i-1}\right)=0$ (so $i-1=0$ ). We can take an adapted to $E$ regular set of parameters $(x, y)$ such that $\mathscr{J}_{P}(\mathscr{D}, E)=(\underline{y}=0)$ and we can suppose that $\mathscr{D}$ is generated at $P$ by

$$
a \partial / \partial x+b \partial / \partial y
$$

with order (b) $\geqslant r+1$ (otherwise, after $(T-1,0)$ we obtain the form I, or $\operatorname{dim} \mathscr{J}_{P_{1}}\left(\mathscr{D}_{1}, E_{1}\right)=0$, or the adapted order drops), so $\mathscr{J}^{r}(a)=(\underline{y}=0)$ and after $(T-1,0)$ one comes to (3.2.4).

(3.3) THEOREM 1. Let $0<i \leqslant l_{P}(\mathscr{D}, E)$ and assume $r=\nu_{P}(\mathscr{D}, E) \geqslant 2$. Then we have that

$$
\operatorname{Inv}\left(S_{P}(\mathscr{D}, E) ; P_{i}\right)<\operatorname{Inv}\left(S_{P}(\mathscr{D}, E) ; P_{i-1}\right)
$$

for the lexicographic order. 
Proof. Because of Lemma $1, \operatorname{dim} \mathscr{J}_{P_{i-1}}\left(\mathscr{D}_{i-1}, E_{i-1}\right)=1$, otherwise $i-1=$ $l_{P}(\mathscr{D}, E)$. If $i=1$ or if $\operatorname{dim} \mathscr{J}_{P_{i}}\left(\mathscr{D}_{i}, E_{i}\right)=0$, the result is trivial. Let us suppose that $i>1$ and that $\operatorname{dim} \mathscr{J}_{P_{i}}\left(\mathscr{D}_{i}, E_{i}\right)=1$. Then $s_{P_{i-1}}\left(E_{i-1}\right) \geqslant 1, s_{P_{i}}\left(E_{i}\right) \geqslant 1$, and $\mathscr{D}_{i-1}$ and $\mathscr{D}_{i}$ have one of the forms I-V.

As a notation let us write for instance III $\rightarrow$ I if $\mathscr{D}_{i-1}$ has the form III at $P_{i-1}$ and $\mathscr{D}_{i}$ has the form I at $P_{i}$. If we have I $\rightarrow$ I, III $\rightarrow$ III, IV $\rightarrow \mathrm{V}, \mathrm{V} \rightarrow \mathrm{V}$, Lemma 2 gives us the result. The only transition beginning at $\mathrm{I}$ is $\mathrm{I} \rightarrow \mathrm{I}$. Because of Lemma 3, there is no transition beginning at II. If III $\rightarrow$ I or III $\rightarrow$ II, the type drops and the transitions III $\rightarrow$ IV and III $\rightarrow$ V are not possible. The only transition beginning at IV is IV $\rightarrow \mathrm{V}$. If $\mathrm{V} \rightarrow \mathrm{I}$ or $\mathrm{V} \rightarrow$ II the type drops, if $\mathrm{V} \rightarrow$ III the stability invariant drops and $\mathrm{V} \rightarrow$ IV is not possible.

(3.4) Note that if $\gamma_{P}(\mathscr{D}, E)<\infty$, then $\gamma_{P}(\mathscr{D}, E) \in(1 / r$ !)N. As the other invariants are nonnegative integers, we have the following:

CoROllary 1. If $\nu_{P}(\mathscr{D}, E) \geqslant 2$, then $S_{P}(\mathscr{D}, E)$ is always finite.

(3.5) As the adapted order is semicontinuous and $\mathscr{D}$ is multiplicatively irreducible and adapted to $E$, the points of adapted order greater or equal to 1 form a finite set of $X$, thus we can state:

COROLlaRY 2 (desingularization theorem). All the possible sequences

$$
X=X_{0} \stackrel{\pi_{0}}{\leftarrow} X_{1} \stackrel{\pi_{1}}{\leftarrow} \cdots
$$

obtained by blowing-up successive points with adapted order for the strict transform of $\mathscr{D}$ greater than or equal to 2 are finite sequences. Thus the strict transform $\left(\mathscr{D}^{\sim}, E^{\sim}\right)$ of $\mathscr{D}$ by the composition of all the blowing-ups of any one of these sequences has only points whose adapted order is less than or equal to 1.

\section{The case of adapted order equal to 1.}

(4.1) LeMMA 4. Let us suppose that $\mathscr{D}$ has the form I at $P$ in the adapted to $E$ regular set of parameters $(x, y)$ and that

$$
\gamma(\mathscr{D}, E ; x, y)>\gamma_{P}(\mathscr{D}, E) .
$$

Then $\gamma_{P}(\mathscr{D}, E) \in \mathbf{N}$ and there is a coordinate change $y_{1}=y+\xi x^{\gamma}, \gamma=\gamma_{P}(\mathscr{D}, E)$, such that $\mathscr{D}$ is prepared at $P$ with respect to $\left(x, y_{1}\right)$ and such that

$$
\gamma\left(\mathscr{D}, E ; x, y_{1}\right)=\gamma_{P}(\mathscr{D}, E) \text {. }
$$

Proof. Following notations as in [4], for a power series $f=\sum_{i, j} f_{i j} x^{i} y^{j}, r \in \mathbf{N}$, and $\gamma \in \mathbf{Q}$, denote

$$
\{f\}_{(x, y)}^{\gamma, r}=\sum_{j=0}^{r} f_{\gamma(r-j), j} x^{\gamma(r-j)} y^{j}
$$

(if $\gamma(r-j) \notin \mathbf{N}$, set $f_{\gamma(r-j), j}=0$ ). Let us suppose that $\mathscr{D}$ is generated at $P$ by

$$
D=a \cdot x \partial / \partial x+b \partial / \partial y \text {, }
$$

where $\mathscr{J}^{r}(b)=(\underline{y}=0), r=\nu_{P}(\mathscr{D}, E)$. Let $\gamma=\gamma(\mathscr{D}, E ; x, y)$ and denote

$$
\{D\}_{(x, y)}=\left((1 / y)\{y a\}_{(x, y)}^{\gamma, r},\{b\}_{(x, y)}^{\gamma, r}\right)
$$


which is an element of $(k[x, y])^{2}$. The fact of $\mathscr{D}$ being prepared with respect to $(x, y)$ only depends on $\{D\}_{(x, y)}$ (modulo $k^{*}=k-\{0\}$ ).

Let $\left(x_{2}, y_{2}\right)$ be an adapted to $E$ regular set of parameters such that $\mathscr{D}$ is prepared with respect to $\left(x_{2}, y_{2}\right)$ and such that

$$
\gamma\left(\mathscr{D}, E ; x_{2}, y_{2}\right)=\gamma_{P}(\mathscr{D}, E) \text {. }
$$

We have that

$$
x_{2}=\lambda \cdot x \cdot u, \quad y_{2}=\mu \cdot y \cdot v+\rho x^{n} \cdot w
$$

where $u, v, w$ are units in $k[[x, y]]=\hat{\mathcal{O}_{X, P}}$ which are equal to 1 modulo the maximal ideal $\hat{m_{P}}$ and $\lambda, \mu \in k^{*}, \rho \in k$. Because of (4.1.1) one has $\rho \neq 0$ and $n=\gamma_{P}(\mathscr{D}, E)$; in particular $\gamma_{P}(\mathscr{D}, E) \in \mathbf{N}$. In fact, looking at the monomial $\sigma \cdot y^{r}, \sigma \in k^{*}$, which occurs in the coefficient $b$, one can see that if $n<\gamma(\mathscr{D}, E ; x, y)$ and $\rho \neq 0$ then

$$
\gamma\left(\mathscr{D}, E ; x_{2}, y_{2}\right)=n
$$

and if $n>\gamma(\mathscr{D}, E ; x, y)$ then

$$
\gamma\left(\mathscr{D}, E ; x_{2}, y_{2}\right) \geqslant \gamma(\mathscr{D}, E ; x, y) .
$$

Consider the coordinate change

$$
x_{1}=x ; \quad y_{1}=y+\left(\rho / \lambda^{n}\right) x^{n} .
$$

Then $\gamma\left(\mathscr{D}, E ; x_{1}, y_{1}\right)=n$ and by an easy computation one has that

$$
\{D\}_{\left(x_{1}, y_{1}\right)}=\mu^{r} \cdot\{D\}_{\left(x_{2}, y_{2}\right)}
$$

thus $\mathscr{D}$ is prepared with respect to $\left(x, y_{1}\right)$.

(4.2) THEOREM 2. If $\nu_{P}(\mathscr{D}, E)=1$, then the stationary way $S_{P}(\mathscr{D}, E)$ is infinite if and only if there is a step $P_{l}, l>1$, such that $\mathscr{D}_{l}$ takes the form $\mathrm{I}$ at $P_{l}$ and $\gamma_{P_{l}}\left(\mathscr{D}_{l}, E_{l}\right)=\infty$. Moreover, if $i \geqslant l$ then $\mathscr{D}_{i}$ takes the form I at $P_{i}$ and $\gamma_{P_{i}}\left(\mathscr{D}_{i}, E_{i}\right)=$ $\infty$.

Proof. If $\gamma_{P_{i}}\left(\mathscr{D}_{i}, E_{i}\right)<\infty$ for all $i=1, \ldots$, then the same techniques as in Theorem 1 show that $S_{P}(\mathscr{D}, E)$ is finite. Thus, for some $l \geqslant 1$ one has that $\gamma_{P_{l}}\left(\mathscr{D}_{l}, E_{l}\right)=\infty$. Because of the multiplicative irreducibility of $\mathscr{D}_{l}, \mathscr{D}_{l}$ takes the form I or II at $P_{l}$. If $\mathscr{D}_{1}$ takes the form II, then Lemma 3 leads us to a contradiction with the fact that $S_{P}(\mathscr{D}, E)$ is infinite, so $\mathscr{D}$, takes the form I. As the only way to lose the form I with the transformation $(T-1,0)$ is to reach $\gamma_{P_{i}}\left(\mathscr{D}_{i}, E_{i}\right)=1$ and in this case $\operatorname{dim} \mathscr{J}_{P_{i}}\left(\mathscr{D}_{i}, E_{i}\right)=0$ (so $S_{P}(\mathscr{D}, E)$ is finite), then the last statement holds.

For the converse, let us suppose that $\mathscr{D}_{l}$ takes the form I at $\mathscr{D}_{l}$ and $\gamma_{P_{l}}\left(\mathscr{D}_{l}, E_{l}\right)=$ $\infty$. It is enough to prove that $l<l_{P}(\mathscr{D}, E)$ and that $\mathscr{D}_{l+1}$ takes the form I at $P_{l+1}$ and $\gamma_{P_{l+1}}\left(\mathscr{D}_{l+1}, E_{l+1}\right)=\infty$. As $\gamma_{P_{l}}\left(\mathscr{D}_{l}, E_{l}\right)=\infty$, we can choose an adapted to $E_{l}$ regular set of parameters $(x, y)$ at $P_{l}$ such that

$$
\gamma\left(\mathscr{D}_{l}, E_{l} ; x, y\right)>2 \text {; }
$$

by applying $(T-1,0)$ we have that

$$
\gamma\left(\mathscr{D}_{l+1}, E_{l+1} ; x^{\prime}, y^{\prime}\right)>1
$$

and then $l<l_{P}(\mathscr{D}, E)$ and $\mathscr{D}_{l+1}$ has the form I. Now, suppose that

$$
\gamma_{P_{l+1}}\left(\mathscr{D}_{l+1}, E_{l+1}\right)=\gamma<\infty \text {. }
$$


Then we can choose $(x, y)$ such that

$$
\gamma\left(\mathscr{D}_{l}, E_{l} ; x, y\right)>\gamma+1,
$$

and by applying $(T-1,0)$ we have that

$$
\gamma\left(\mathscr{D}_{l+1}, E_{l+1} ; x^{\prime}, y^{\prime}\right)>\gamma .
$$

By Lemma 4, there is a coordinate change $x_{1}^{\prime}=x^{\prime}, y_{1}^{\prime}=y^{\prime}+\xi x^{\prime \gamma}$ such that $\mathscr{D}_{1+1}$ is prepared with respect to $\left(x_{1}^{\prime}, y_{1}^{\prime}\right)$ and

$$
\gamma\left(\mathscr{D}_{l+1}, E_{l+1} ; x_{1}^{\prime}, y_{1}^{\prime}\right)=\gamma .
$$

Consider the change $x_{1}=x, y_{1}=y+\xi x^{\gamma+1}$. Then by (3.1.2) and the argument following (3.1.2) we have that

$$
\gamma\left(\mathscr{D}_{l}, E_{l} ; x_{1}, y_{1}\right)=\gamma+1<\infty
$$

and $\mathscr{D}_{1}$ is prepared with respect to $\left(x_{1}, y_{1}\right)$, which is a contradiction with the fact that $\gamma_{P_{l}}\left(\mathscr{D}_{l}, E_{l}\right)=\infty$. Then

$$
\gamma_{P_{l+1}}\left(\mathscr{D}_{l+1}, E_{l+1}\right)=\infty \text {. }
$$

(4.3) Remark. As a corollary of Theorem 2 one can easily obtain the result of Seidenberg [6, Theorem 12].

(4.4) Let us suppose that $\mathscr{D}$ takes the form I at $P$ and that $\gamma_{P}(\mathscr{D}, E)=\infty$. Then there are two possibilities:

A. There is an adapted to $E$ regular set of parameters $(x, y)$ such that $\mathscr{D}$ is prepared with respect to $(x, y)$.

B. There is not such an adapted regular set of parameters.

In case $\mathrm{A}, \gamma(\mathscr{D}, E ; x, y)=\infty$ and $\mathscr{D}$ is generated at $P$ by

$$
D=a x \partial / \partial x+(y \cdot u) \partial / \partial y,
$$

where $u$ is a unit in $\mathcal{O}_{X, P}$. Take the irreducible closed curve $Y$ of $X$ given at $P$ by $y=0$. One can desingularize $Y$ by a finite number of blowing-ups in closed points different from $P$, and making more blowing-ups, if necessary, one can suppose that $Y$ has normal crossings with $E$. Now, if one makes the blowing-up of $X$ with center at $Y$, it has the only effect of adding $Y$ to $E$ and thus in view of (4.4.1) the adapted order at $P$ comes to 0 . In this case we have an "algebraic resolution" at $P$.

If case B holds, let $X^{\wedge}$ be the formal scheme of $X$ at $P$ and let $\mathscr{D}^{\wedge}$ be obtained from the morphism $X^{\wedge} \rightarrow X$ in a way similar to $\mathscr{D}^{\pi}$. If $(x, y)$ is an adapted to $E$ regular set of parameters in which $\mathscr{D}$ takes the form $I$, then the infinite sequence of preparations coordinate changes $y \mapsto y_{1}$ is always convergent in $\mathcal{O}_{X, P}=\mathcal{O}_{X, P}$ and leads us to a regular set of parameters $\left(x, y^{\wedge}\right)$ of the maximal ideal of $\mathcal{O}_{X, P}$ which is adapted to $E^{\wedge}$ ( = inverse image in $X^{\wedge}$ of $E$ ) and such that

$$
\gamma\left(\mathscr{D}^{\wedge}, E^{\wedge} ; x, y^{\wedge}\right)=\infty \text {. }
$$

If $Y^{\wedge}$ is given by $y^{\wedge}=0$, then the blowing-up of $X^{\wedge}$ with center at $Y^{\wedge}$ adds $Y^{\wedge}$ to $E^{\wedge}$ and then the adapted to $E^{\wedge} \cup Y^{\wedge}$ order of $\mathscr{D}^{\wedge}$ at $P$ is 0 . One has an "algebroid resolution". 
(4.5) ExAmple. Case B effectively occurs as the following example shows: $X=$ $\mathbf{A}^{2}(k), P=$ origin and $\mathscr{D}$ is globally generated by

$$
D=x \cdot x \partial / \partial x+(y+x) \partial / \partial y,
$$

where $E$ is given by $x=0$.

In fact, let us suppose that there exists a change $y \mapsto y_{1}, y_{1} \in \mathcal{O}_{X, P}$ such that, locally at $P$,

$$
D=x \cdot x \partial / \partial x+\left(u \cdot y_{1}\right) \partial / \partial y
$$

where $u$ is a unit in $\mathcal{O}_{X, P}$. Then the sequence of the infinitely near points obtained by blowing-up $y_{1}=0$ at the origin is necessarily equal to $S_{P}(\mathscr{D}, E)$. By computing $S_{P}(\mathscr{D}, E)$ from (4.5.1) one obtains that $y_{1}=0$ defines the algebroid curve

$$
y=-\sum_{i>1}(i-1) ! x^{i}
$$

which is not an algebraic curve.

\section{REFERENCES}

1. F. Cano, Teoria de distribuciones sobre variedades algebraicas, Colecc. de Mon. del Instituto Jorge Juan, C.S.I.C., Madrid, 1983.

2. J. Giraud, Forme normale d'une fonction sur une surface de caractéristique positive, Bull. Soc. Math. France 111 (1983), 109-124.

3. Condition de Jung pour les revêtements radiciels de hauteur 1, Proc. Algebraic Geometry, Tokyo/Kyoto 1982, Lecture Notes in Math., vol. 1016, Springer-Verlag, 1983, pp. 313-333.

4. H. Hironaka, Desingularization of excellent surfaces, Advanced Science Seminar in Algebraic Geometry (Summer 1967), Mimeographed notes by B. Benett, Bowdoin College.

5. Characteristic polyhedra of singularities, J. Math. Kyoto Univ. 10 (1967), 251-293.

6. A. Seidenberg, Reduction of singularities of the differential equation $A d y=B d x$. Amer. J. Math. 90 (1968), 248-269.

7. A. van den Essen, Reduction of singularities of the differential equation $A d y=B d x$, Equations Differentielles et Systèmes de Pfafl dans le Champ Complexe, Lecture Notes in Math., vol. 712, Springer-Verlag, 1979, pp. 44-49.

Departamento Algebra y Fundamentos, Facultad de Ciencias, Universidad de Valladolid, VAILADOLID, SPAIN

Current address: Departamento de Algebra y Geometria, Facultad de Ciencias, Valladolid 47005, Spain 